\title{
EL ALCANCE DE LA MICROECONOMIA
}

Mis primeros pasos de iniciación en el conocimiento de la microeconomía fueron dados bajo la dirección atenta del Dr. Uros Bacic. Tuve el orgullo de colaborar en el dictado de su curso Teoría de los Precios y aún hoy después de su desaparición física, sigue siendo un modelo en mi apego a la teoría microeconómica. Su pensamiento dotado de permanente renovación y de inclinación al estudio de nuevas ideas, constituye el marco para las siguientes reflexiones.

La mayoría de los textos de microeconomía contienen la base necesaria para el estudio de los bienes, consumidores, productores, los resultados clásicos de la existencia de equilibrio general y la teoría del bienestar dentro de un marco de asignación de recursos escasos entre fines alternativos.

Tradicionalmente se asocia a la microeconomía con individuos mientras que la macroeconomía trata de conceptos agregados como el nivel de empleo, producción y precios. Sin embargo los intereses actuales se han trasladado. Los temas que más captan la atención dentro de la microeconomía, se refieren a los problemas de asignación para la sociedad en su conjunto. Los desarrollos recientes han sobrepasado los estrechos limites planteados incialmente para la microeconomía.

Muchos de estos avances han surgido de descontentos con supuestos y el análisis subyacente de la teoría tradicional y llevan, a mi entender, a una nueva definición del alcance de la microeconomía, como así también a una ampliación de su campo.

\section{LOS SUPUESTOS}

Se ha ligado el análisis económico al uso de ciertos componentes claves encuadrados por algunos autores (Holton, 1992) en la tradición del liberalismo económico, que representa una amalgama de ideas tomadas de varias fuentes, Adam Smith, la escuela neoclásica y teóricos tales como Milton Friedman de la escuela de Chicago. Es quizás más adecuado considerar a los supuestos del análisis como parte del individualismo metodológico. Estos conceptos claves son: soberanía individual, propio interés, racionalidad, derechos de propiedad privados, autoregulación de los mercados y orden espontáneo.El individuo es el juez final de las decisiones o las elecciones sobre su propio bienestar y las acciones necesarias para lograrlo. Tiene capacidad de ejercer su soberanía individual y está motivado para hacerlo por propio interés.

Habiendo partido del individuo pre-social que cuenta con la capacidad de actuar de una manera soberana, guiado por la racionalidad y persiguiendo su propio interés, se hace un puente entre los atributos pre-sociales y la vida social a través de instituciones claves de derechos de propiedad privada y mercados autoregulados. El punto esencial de esos derechos privados es que permiten a los individuos de- 
fender su propio interés. En este contexto el mercado es el sistema que permite la libertad humana, regulándose a través del sistema de precios. El análisis de determinación de precios dentro de las relaciones de mercado fue desarrollado con alto grado de sofisticación.

Los individuos no guiados por el bienestar de la sociedad ni con un plan general en el que tengan roles predeterminados toman decisiones económicas que no conducen al caos.

En este esquema surgen elementos de controversia que en general han servido para ampliar más que a restringir el campo de la microeconomía.

\section{LA RACIONALIDAD}

El uso frecuente del comportamiento racional en la teoría es presentado como un término instrumental y por lo tanto más científico que moral, lo que plantea paralelamente un traslado en la economía de lo político, moral o normativo a lo científico e instrumental con la formalización matemática de una serie de relaciones.

No es fácil definir con exactitud el comportamiento racional.La racionalidad se puede analizar en varios sentidos y a veces contradictorios. Actuar racionalmente puede referirse a acciones técnicamente efectivas en asegurar objetivos de propio interés de los actores dadas las elecciones abiertas a ellos. Un ejemplo típico es "Dado un aumento de precios es racional utilizar un artículo que sea más barato". Sin embargo, actuar racionalmente significaría que los agentes podrían dar una razón plausible de por qué actúan de esta manera, sin que necesariamente involucre la eficiencia técnica: "Dadas las recomendaciones en cuanto al deterioro de la capa de ozono, sólo es racional invertir en productos que eviten tal daño" o "Dado que por varias generaciones una familia ha usado gas para cocinar y calentarse, es racional que una persona perteneciente a esa familia quiera instalar gas en su nuevo hogar". En el primer ejemplo se asocia racionalidad con soluciones técnicamente eficientes a cuestiones de cómo asegurar mejor el propio interés, mientras que en la segunda serie de ejemplos se asocia racionalidad a valores del entorno o a tradiciones. En el mundo de la microeconomía tradicional se ha definido a la racionalidad en términos del primer tipo, más que de la segunda forma, pasando a ser un concepto instrumental.

El interés en este concepto nace de dos motivaciones diferentes. Existe, por un lado, un deseo de conocer el uso de modelos que incluyen el comportamiento racional para explicar y predecir el comportamiento real. Esta motivación subyace en la teoría del equilibrio general. A pesar de ser un concepto de contenido neutral en el sentido ético, es razonable suponer que el comportamiento sea racional a pesar de que el comportamiento real pueda tomar cualquier forma. En este sentido en la observación de la realidad se aprecian indices de racionalidad en el comportamiento de los individuos y paralelamente se pueden observar irracionalidades que tienen explicaciones convincentes.

En cuanto a la posibilidad de explicar comportamientos reales, la forma corriente de maximización de objetivos, ha sufrido una serie de críticas, basadas en que los hechos humanos tienen otras motivaciones entre las que se incluye el propio interés (Sen, 1990). Cuando los individuos no actúan solos sino dentro de grupos puede ser que exista un énfasis en otros valores que el propio interés (Akerlof, 
1984), argumentándose que el éxito económico se ha logrado más plenamente en culturas que tienen normas de conducta y valores diferentes a la búsqueda del propio interés tales como la "ética japonesa" (Sen, 1990). La racionalidad instrumental se refiere al comportamiento pero no define el contenido de beneficios y costos, que forman parte de la subjetividad de los individuos. La ampliación de la función de utilidad a optimizar, no invalida el concepto, sino que lo trata de forma diferente.

Por otra parte, como si fuera un ejercicio económico de perspectiva es interesante saber cómo el individuo se debería comportar racionalmente en una situación dada. En este caso podría ser presentado bajo términos no éticos sino sólo como lo que se debería hacer para promover su propio interés (Harsanyi, 1977). Sin embargo podría constituir un término de contenido moral si la racionalidad se vuelve un fin en sí mismo. Es no sólo eficiente ser racional sino también deseable (Holton, 1992), lo que evidentemente situaría el concepto en el campo de la economía normativa.

Se ha enfatizado también que los individuos pueden no maximizar ninguna función en especial y el agente decisor puede tener una conducta que busque un resultado aceptable o satisfactorio, con un enfoque de "racionalidad limitada" en situaciones de información parcial (Simon, 1982). La información necesaria en un mundo imperfecto aumenta los costos de obtenerla teniendo en cuenta el fenómeno de interdependencia, lo que hace necesario conocer el comportamiento de otros agentes. $\mathrm{Si}$ algunos individuos tienen poder sobre otros y conocen información que otros no poseen, se presentan problemas de "información asimétrica". En este sentido la principal implicación del uso del concepto de racionalidad es el exceso de información necesaria, incompatible con los limites humanos, lo que conduce no a la destrucción del concepto de racionalidad sino al enfoque Simon.

Se puede argumentar también que la racionalidad no es un principio esencial para una teoría en economía y que su poder y utilidad deriva no de su consideración aislada, sino de su conjunción con otros conceptos básicos de la teoría. Esta visión (Arrow, 1990) surge del conocimiento de que para hacer de la racionalidad un concepto útil se deben hacer supuestos adicionales fuertes. Es así que para que la agregación de las demandas resulte también en un comportamiento racional se deben realizar supuestos tan fuertes como los de igualdad de los gustos en el sentido de igualdad de funciones de utilidad o de homotecia. En ese marco Arrow considera que el concepto implica que el individuo requiere de una gran capacidad para procesar información y realizar el cálculo, lo que es incompatible con los límites del individuo. Esto plantea, por una parte, el mismo problema de Simon y paralelamente se reconoce que las decisiones en un marco real no puede plantearse únicamente a través del concepto de racionalidad instrumental.

Un elemento interesante del criticismo a la racionalidad es que el cálculo en términos de maximización de intereses individuales deja de lado el conflicto entre clases sociales (Hollis y Nell, 1975). Es conveniente observar con cuidado tal afirmación. El análisis se realiza en términos de colectivos y no de individuos y toma en cuenta sólo el concepto de racionalidad instrumental. Las ideologias, los mitos, los dogmas no importan, y los mercados eficientes caracterizan a la sociedad. Pero en el mundo real los agentes tienen información incompleta y capacidad mental limitada con la que procesar la información. Por lo tanto se desarrollan 
reglas y normas para estructurar el intercambio. El orden social se logra como resultado de un proceso de conflicto entre las partes interesadas, con un papel destacado de las instituciones.

\section{LAS INSTITUCIONES}

En la microeconomía tradicional, las instituciones no existen o en su defecto se urge a una reforma de ellas, argumentando que representan los mayores obstáculos para el funcionamiento de los mercados. El concepto de racionalidad instrumental hace innecesarias las instituciones. Sin embargo para realizar un tratamiento más adecuado del tema, es conveniente realizar algunas aclaraciones. Las instituciones pueden surgir de la acción espontánea de los agentes, cuyo caso más prominente es el mercado. En este sentido se pueden considerar dos casos:

* intercambio entre dos personas: un consumidor A puede intercambiar unidades del bien X por unidades del bien $\mathrm{Y}$ con el consumidor $\mathrm{B}$.

* mercados: donde el intercambio se produce entre más de dos agentes. Estos mercados pueden estar abiertos para todos los agentes o sólo para algunos agentes específicos.

Otra clase de instituciones que pueden surgir espontáneamente son las normas sociales y la tradición. En ese contexto la familia (no como consumidor individual) y otros pequeños grupos sociales resultan las más importantes instituciones.

En las instituciones conscientemente diseñadas por la acción humana el gobierno aparece como la más destacada. Si se la une a los mercados, la actividad privada pura, en ausencia de gobierno logra para los participantes beneficios mutuos a través del intercambio. Sin embargo esto presupone que las únicas alternativas para los individuos son intercambiar los bienes o no hacerlo. No se plantea la elección del robo de los bienes propiedad del otro individuo. Surge así la necesidad de un contrato constitucional que establezca los derechos de propiedad como así también procedimientos de refuerzo de tales derechos (Mueller, 1989)(1)

Dentro del gobierno como institución política es posible diferenciar:

* Dictador: un agente toma las decisiones y por ejemplo decide el monto de bienes a ser asignados a cada uno de los agentes.

* Democracia: como caso extremo del anterior, la asignación podría ser votada. Esto conduce al estudio de las reglas de decisión social.

Además del gobierno, pueden surgir otras instituciones

públicas y privadas que corrigen las anomalias individuales y mitigan sus efectos (Frey y Eichenberger, 1991)

Si además de lo anterior se tiene en cuenta la producción en el sentido de la elección de transformaciones técnicamente factibles de insumos en productos otras instituciones (Blad y Keiding, 1990) serían:

* Economía centralmente planificada, en la que una agencia fija precios y cantidades de acuerdo a los objetivos previamente fijados en un plan.

* Economía perfectamente competitiva: los precios están dados por el mercado y los productores eligen sus planes de producción a esos precios y los consumidores eligen los intercambios a esos precios dados.

Una interesante descripción de las instituciones económicas del capitalismo 
se encuentra en el enfoque sobre las restricciones a las que llegan voluntariamente los que efectúan transacciones, cuando son libres de imponer las limitaciones sobre ellos mismos (Williamson, 1985). Esto permite la comprensión de coaliciones, instituciones y estructuras de contrato que incluyen la asignación y la división de los derechos de propiedad y de las instituciones que establecen y mantienen un comportamiento de tipo contractual. Las instituciones del capitalismo son en este caso más que las firmas, los mercados y los contratos de relación. Existe una interdependencia entre los actores que adopta formas muy complejas y que motiva toda una serie de arreglos: joint-ventures, mutuales, clubes, cooperativas, familias. Esta ampliación del tratamiento microeconómico tradicional abarca entonces los más diversos aspectos del proceso de elección.

Paralelamente a los problemas de existencia de las instituciones, se plantea la estabilidad de las mismas, tal como se hace tradicionalmente con el equilibrio de mercado. Si por ejemplo uno de los agentes de una economía perfectamente competitiva se da cuenta de que es el único oferente, esto lo inducirá a usar la situación de manera de obtener más beneficios, pero no habrá seguido las reglas (en cuanto a elegir la producción que haga máximos sus beneficios a los precios dados). Esto convertiría en inestable a la institución economía perfectamente competitiva. El diseño de instituciones para asegurar el comportamiento económico dentro de los límites dados por la institución se denomina implementación (Hurwicz, 1972).

\section{LA COMPETENCIA}

En este aspecto es útil considerar tres aspectos del tema:

1) La firma competitiva: la competencia perfecta del modelo tradicional cuenta con la ausencia de competencia en términos de lo que se considera rivalidad en el logro de un resultado exclusivo. Con respecto a esto también es conveniente ampliar el modelo tradicional.

Se debe hacer una diferenciación entre competencia y los objetivos de los hombres de negocios." En la vida económica la competencia no es un objetivo, es un medio de organizar la actividad económica para lograr un objetivo" (Stigler, 1968). Sería útil distinguir como primer paso, entre competencia inestructurada y estructurada. La primera es coherente con la visión tradicional de competencia, donde la empresa tiene poca o nula elección. Deben obedecer la ley de mercado o salir de él. La segunda se encuentra en la mayoría de los mercados, donde un número estrictamente limitado de firmas son capaces de determinar la extensión y la forma de la competencia ejerciendo una medida sustancial de elección sobre cómo deben comportarse. Es conveniente tener en cuenta aquí el concepto de estrategia de negocios (Porter, 1980), y la ampliación de los objetivos de las empresas ( Baumol, 1959) que en conjunto pueden resultar en comportamientos anticompetitivos. Una estrategia puede ser defensiva $u$ ofensiva. En el primer caso, el agente evita riesgos y protege una situación que considera privilegiada, con un comportamiento que puede ser definido como cauto y resistente al cambio e involucra a veces la cooperación y la colusión con los otros.

Gradualmente, los trabajos de investigación han comenzado a abordar cuestiones 
tales como los procesos de decisión dentro de la firma y la consideración de las relaciones entre los diferentes grupos de una organización.

2) Normatividad de la competencia: se supone que bajo competencia perfecta, ninguno de los individuos tiene suficiente control sobre el mercado para influenciar las acciones de otros agentes. Las ventajas crecerían para quien se comporta más racional o eficientemente que los otros. Sin embargo el acceso a la racionalidad y eficiencia estará disponible para todos. El supuesto de que los mercados trabajan bien si no están afectados por restricciones externas y que el aumento de la competitividad mejora su desempeño, se deriva del análisis de competencia de precios en los mecados de bienes privados.

Si la competencia consume recursos como en todos los modos de competencia no basados en el precio, una mayor competencia no necesariamente mejora el bienestar social. Sin embargo la eficiencia no puede ser evaluada únicamente por el resultado, ya que las acciones de los individuos pueden no ser las proyectadas, sino que debe ser juzgada como el proceso del cual surgen las decisiones individuales.

Cuando el marco institucional impide que las acciones individuales puedan ser normativamente correctas se plantea el concepto de búsqueda de rentas, las que existen donde las asimetrías impiden el flujo de recursos, tanto en los mercados políticos, como en los de bienes, factores y activos. La búsqueda de rentas es un tema reciente en el análisis de los mercados, relacionado también al marco institucional. (Mueller, 1990).

3) Equilibrio competitivo general: la teoría del equilibrio general competitivo (Arrow y Debreu, 1959) explica como economías descentralizadas, con individuos persiguiendo su propio interés, coordinan sus decisiones de manera de lograr el equilibrio, confiando sólo en las señales de precios. Sin embargo el mercado con precios perfectamente flexibles es más bien una excepción en la realidad. Los precios son fijos o tienen ciertas rigideces para su aumento o disminución. Bajo estas condiciones, se enuncian modelos de asignación que proveen también un esquema teórico para tratar la macroeconomía keynesiana (Drèze, 1975; Blad y Keiding, 1990).

Los comportamientos no competitivos ( competencia imperfecta), se han considerado también a partir de la teoria del equilibrio general, obteniéndose ideas que no se hallan en los modelos de equilibrio parcial (Shapley y Shubick, 1977).

\section{LA INCERTIDUMBRE}

La incertidumbre es un rasgo de la vida de los individuos. Se refiere a una inevitable ignorancia del futuro. Los modelos económicos discutidos por la microeconomía tradicional consideran que los agentes conocen con certidumbre todo lo relevante a sus decisiones. Esto parece conducir a un modelo sin sentido ya que no hay manera de tomar decisiones ahora suponiendo conocer todos los posibles usos de los recursos en el futuro.

Se ha hecho un tratamiento formal de la incertidumbre usando teoría de probabilidades. La teoría de la utilidd esperada subjetiva provee una descripción lógica de lo que debería hacer el individuo racional en un mundo con incertidumbre. La utilidad esperada subjetiva ha tratado los temas del impacto de la incertidumbre 
sobre el comportamiento de individuos y mercados. Siguiendo los trabajos de Ramsey (1926), Savage (1954) y Anscombe y Aumann (1963), la incertidumbre puede ser descripta por probabilidades subjetivas, basadas en el análisis de árboles de decisión de acuerdo a Raiffa (1968). Estudios recientes, combinan el estudio de las formas clásicas de evitar el riesgo, tales como los seguros, junto a problemas de información asimétrica, azar moral y seleción adversa (Rees, 1989). Esta teoría ha sido tradicionalmente de mayor aplicación en modelos de equilibrio parcial, aún cuando existen extensiones al campo del equilibrio general.

Otro tratamiento de la incertidumbre introduciendo bienes contingentes involucran problemas de información asimétrica. La teoría del equilibrio general competitivo fue desarrollada originalmente considerando la inexistencia de incertidumbre. Los bienes contingentes (Debreu, 1953; Arrow, 1964) en cuya descripción se incluye un cierto evento que debe ocurrir para que tenga lugar el libramiento, capturan los elementos esenciales de la incertidumbre. La idea es agregar el evento ambiente en el cual el bien está disponible. Con certidumbre el bien está especificado por sus características físicas, la localización y la fecha de su disponibilidad. Pero tal bien será diferente si se producen diferentes condiciones de ambiente. La incertidumbre es la disponibilidad de los bienes que es contingente con la ocurrencia de algunos eventos del ambiente. Con esto el modelo es muy similar al de equilibrio general competitivo y no se introdujo el concepto de probabilidad. La información, con un rol importante en la distinción entre los estados de la naturaleza, aparece nuevamente como el elemento al cual dedicarle las energías en la investigación. Las diferencias en la información de los agentes, junto a un tratamiento unificado y más formal del tiempo y la incertidumbre usando bienes contingentes constituye un importante avance en el análisis (Radner, 1972)

\section{CONCLUSION}

El estudio de algunos de los análisis más trascendentes de la microeconomía pone de relieve que el alcance de la teoría es más amplio que lo considerado tradicionalmente. Los problemas de información y conflicto aparecen como subyacentes a todos los temas tratados. Esto conduce al pensamiento de que las cuestiones de interacción entre los agentes individuales y las instituciones juegan un rol central.

Desde este punto de vista es quizás más conveniente pensar a la microeconomía como " el análisis de la interacción entre agentes en diferentes contextos institucionales" (Blad y Keiding, 1990). Cuando se percibe esto, nos damos cuenta que se debe enseñar a los estudiantes de microeconomía mucho más de lo que se pensaba inicialmente.

Alicia B. Giacchero

Departamento de Economía Universidad Nacional del Sur

NOTAS

1. Los artículos pioneros fueron: Tullock. Gordon (1967) The Welfare Costs on Tariffs, Monopolies and Theft. 
Western Economic Journal, Junio, p.224-32 y Krueger, Ann (1974), The Political economy of the Rent Seeking Society, American Economic Review, June, p.291-303. Ambos artículos están reimpresos en el siguiente libro que contiene las principales aportaciones iniciales al tema: Buchanan, J., Tollison, R. y Tullock, G. (eds.) (1980) Towards a Theory of the Rent Seeking Society, College Station. Texas A\&M Press. Una recopilación de los últimos aportes principales se encuentra en : Rowley, Ch., Tollison, R. y Tullock, G. (eds.) (1987) The Political Economy of Rent Seeking. Boston, Dordrecht, Lancaster: Kluwer Academic Publisher

\section{REFERENCIAS}

AKERLOF.G. (1984) An Economic Theorist's Book of Tales. Cambridge University Press. Cambridge ANSCOMBE. F.J. Y AUMANN, R. (1963) A definition of subjective probability. Annals of Mathematical Statistics, 34

ARROW. K. (1964) The role of securities in the optimal allocation in risk bearing. Review of Economic Studies

ARROW. K. (1984) The Economics of Agency. Technical Report Nro. 451 Centre for Research on Organisational Efficiency. Stanford University

ARROW, K. (1990) Economic theory and the hypothesis of rationality en J. Eatwell, M.Milgate y P. Neuman (eds.). Utility and Probability. Macmillan. London.

ARROW, K. Y DEBREU, G. (1959) Existence of equilibrium for a competitive economy. Econometrica, 22. BAUMOL. W. (1959) Business Behaviour. Value and Growth. Macmillan. London

BLAD, M. Y KEIDING, H. (1990) Microeconomics. Institutions, Equilibrium and Optimality. North Holland. Amsterdam

BUCHANAN, J. Y TULLOCK, G. (1962) The Calculus of Consent. University of Michigan Press.

DEBREU. G. (1953) Une économie de l'incertain. Paris. Electricité de France.

DEBREU. G. (1959) Theory of Value. Wiley. New York.

DOWNS, A. (1957) An Economic Theory of Democracy. Harper. New York

DREZE, J. (1975) Existence of exchange equilibrium under price rigidities. International Economic Review. 16.

FREY, B. Y EICHENBERGER, R. (1991) Anomalies in Political Economy. Public Choice, 68.

HARSANYI. J. (1977) Rational Behaviour and Bargaining Equilibrium in Games in Social Situations. Cam bridge University Press.

HOLLIS, M. Y NELL, E. (1975) Rational Economic Man. Cambridge University Press.

HOLTON. R. (1992) Economy and Society. Routledge. London

HURWICZ L. (1972) On informationally decentralized systems en C. Mc Guire y R. Radner (eds) Decision and Organisation. North Holland.

MUELLER, D. (1989) Public Choice 11. Cambridge University Press.

PORTER, M. (1980) Competitive Strategy. Techniques for Analysing Industries and Companies. Free Press. New York.

RADNER. R. (1972) Existence of equilibrium of plans, price and price expectations in a sequence of markets.

Econometrica, 40

RAIFFA. H. (1968) Decision Analysis: Introductory Lectures on Choices under Uncertainty. Reading Mass. Addison-Wesley

RAMSEY. F.P. (1926) Truth and Probability. In F.P.Ramsey, The Foundations of Mathematics and Other Logical Essays, ed. R. Braithwaite, New York: Humanities Press, 1950.

REES, R. (1989) Uncertainty, Information and Insurance en J. Hey (ed) Current Issues in Microeconomics. Macmillan.London.

SAVAGE. L.J. (1954) The Foundations of Statistics. New York. John Wiley

SEN, A. (1990) Rational behaviour en J. Eatwell. M. Milgate y P. Newman (eds) Utility and Probability. Macmillan. London.

SHAPLEY L. Y SHUBIK M. (1977) Trade using one commodity as a mean of payment. Journal of Political Economy. 85.

SIMON, H. (1982) Models of Bounded Rationality. Cambridge Press. MIT.

STIGLER. G. (1968) The Organisation of Industry. Richard Irvin: New York.

WILLIAMSON. O. (1985) The Economics Institutions of Capitalism: Firms. Markets. Relational Contracting. Free Press. Macmillan. London. 